\title{
Importance of Screening for Wellbeing in Diabetes Management
}

\author{
Gunjan Y Trivedi* \\ Cofounder, Society for Energy \& Emotions, Wellness Space, India
}

Submission: July 16 2019; Published: August 09, 2019

*Corresponding author: Gunjan Y Trivedi, Cofounder, Society for Energy \& Emotions, Wellness Space, 119c Swastik Society, Navrangpura, Ahmedabad 380 009, Gujarat, India

\section{Abstract}

Diabetes (Type 1 and 2) is a chronic metabolic disorder impacting both the mind and the body of the individual. The impact on the mind includes emotional, social, cognitive and psychosocial wellbeing of the individual. Thus, diabetes management must not only focus on the impact of the disease on the body but also understand its cognitive, psychological and lifestyle implications.

Given the negative impact of the stress on both the mind and the body, stress screening and management must be incorporated in diabetes management. This review explores stress, its impact on physical and psychological wellbeing in the context of diabetes management. Evidence is presented on the challenges related to measurement of stress and its negative impact on the wellbeing of the individual. Based on the comparison (complexity, scalability, etc.) between measurement of stress and wellbeing, the review highlights the importance of screening for wellbeing using simple psychological wellbeing measurement tool (WHO-5) and its usefulness in providing the framework for assessment of conditions such as stress, depression, etc.

Keywords: Cardiovascular disease; Respiratory disease; Cancer; Eating habits; Physical inactivity; Central obesity; Family history; Alcohol; Tobacco consumption; Emotional stress

\section{Introduction}

In the $21^{\text {st }}$ century, the focus on disease has shifted from infectious diseases to non-communicable diseases (NCDs) such as diabetes, cardiovascular disease, respiratory disease, cancer etc. due to increase in mortality from NCDs (also referred to as chronic diseases) as compared to infectious disease [1]. Lifestyle choices such as poor eating habits, physical inactivity, central obesity, family history, alcohol and tobacco consumption etc. are the key risk factors for chronic diseases including diabetes [2]. Chronic diseases claim anywhere from 65 to $70 \%$ of all deaths globally. Such disease conditions are often connected with psychosocial stress, emotional challenges and cognitive decline. Studies have estimated that average 9 to $23 \%$ of the individuals who had one or more chronic physical disease had comorbid depression (based on assessment of 245,000 participants from 60 countries) [3]. Studies have also validated the probability of having depression is significantly higher in individuals with chronic disease as compared to those who had no chronic physical disease. Recent evidence also highlights the role of stress in chronic disease (this could be due to environment or emotional stress or even poor- quality sleep) [4]. This review focuses on diabetes, stress and the need for measurement of stress and the wellbeing (which is negatively impacted by presence of chronic disease conditions and stress).

\section{Diabetes and Stress - Evidence and Implications}

For diabetes, the scientific evidence confirms the impact of stress on the onset and also on glycaemic control and quality of life [5-8]. Diabetes, similar to other chronic disease, also impacts the emotional health of the individuals (an individual with diabetes is twice as likely to have depression compared to normal healthy controls) [9]. This indicates a two-directional relationship between diabetes and stress. As a result, identification of acute or chronic stress in high risk individuals (such as those without diabetes but with metabolic syndrome components) or in individuals with diabetes (and its complications) is very important. Following sections briefly define key components of stress, how it impacts wellbeing, cognitive functions and challenges involved in the measurement of stress and wellbeing. 


\section{Current Research in Diabetes \& Obesity Journal}

\section{Stress and Wellbeing}

Stress (a nonspecific response of the body to any demand for change) has two components (1) the causes such as environmental stress or life events or lifestyle choices such as poor sleep or too much work related burn-out and (b) the effects - subjective reaction of stress that may include assessment (based on individual's perception) and emotional response [10]. In other words, stress, its causes and impact on each individual is complex and involves understanding of multiple situations and parameters. For the purpose of this review, it is clear that stress measurement involves understanding of the environment (or stimulus as perceived by the individual), individual's own stress response mechanism \& perception/interpretation and finally the biological impact on the physiology and cognitive functions (including sleep quality, memory, focus, etc.). Additional factors to consider include whether the stress is acute or chronic, individual's coping mechanism, social support, etc.

Wellbeing is an important element of health since physical, mental and social wellbeing together constitute good health [11]. This review focuses on "wellbeing" as compared to the Quality of Life (QoL) since QoL is a more comprehensive attempt to objectively understand the quality of life made up of subjective wellbeing of various aspects of life such as physical, social, emotional and so on $[12,13]$.

Any form of stress, chronic diseases such as diabetes or its complications negatively impacts wellbeing in multiple aspects of life resulting in reduced QoL [14]. While the quality of life declines due to presence of chronic disease, the life expectancy continues to increase even in chronic disease conditions because of better education, self-management tools, quality of. Hence, for better management of disease and quality of life, it is important to identify the prevalence of stress and how it impacts its individual.

\section{Measurement of Stress and Wellbeing}

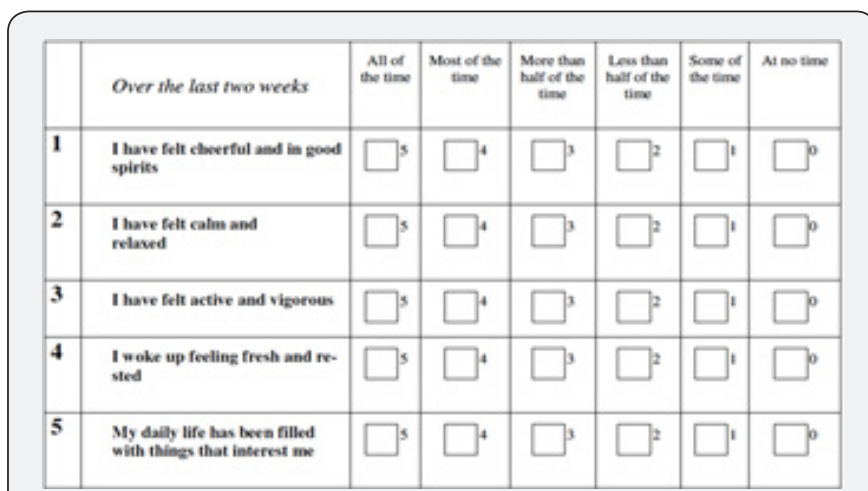

Figure 1: WHO-5 Wellbeing Index questions [18]

Note: The raw score is calculated by totaling the figures of the five answers. The raw score ranges from 0 to 25,0 representing worst possible and 25 representing best possible quality of life. To obtain a percentage score ranging from 0 to 100 , the raw score is multiplied by 4 . A percentage score of 0 represents worst possible, whereas a score of 100 represents best possible quality of life [18].
Measurement of stress is a complex process, however, as discussed earlier, it can be simplified into three steps (1) Understanding of the environment which triggers the stress such as job, marital status, environmental risks such as poor air quality of noisy neighbourhood (2) Measurement of how the individual perceives stress such as psychological stress measure (Psychological Stress Measure or PSM-9 tool, as an example) and (3) physiological measurement of stress through pathophysiological changes including how the stress impacts cognitive functions (brain), body or central/peripheral nervous system [15-17].

For measurement of wellbeing, many tools are available such as WHO-5 wellbeing index by World Health Organization or General Health Questionnaire-GHQ-12 questionnaire [18,19]. This review focuses on WHO-5 (Figure 1) due to its simplicity and broad-based usage in many disease conditions [20]. Several studies on diabetes subjects using WHO-5 have confirmed the usefulness of this and its ease of use for both type 1 and type 2 diabetes [21-23].

For physicians or healthcare professionals, there are two possibilities (1) measure stress, its causes and subjective impact and deal with the complexity or (2) measure subjective wellbeing to screen the individuals needing further attention (e.g. to measure depression or presence of stressors after initial screening). The review of evidence indicates that WHO-5 is a useful first step towards identifying any potential wellbeing challenges and based on the outcome further follow-up studies such as measurement of depression or stress can be pursued [24]. For example, it is possible to pursue evaluation for depression based on a cut-off score of WHO-5 and this would make it easier to save time while obtaining useful insights about the wellbeing with awareness that increased stress of any kind would negatively impact the wellbeing. Diabetes studies comparing depression inventory and WHO-5 have also validated this (e.g. WHO- 5 score of $<13$ or $52 \%$ is likely to indicate the need for depression screening) and the finding consistent with WHO recommendations [20].

\section{Discussions}

The review of evidence confirms the impact of stress as a risk factor for diabetes and for the management of diabetes and its complications. Stress is a complex concept and is difficult to identify, validate and address. Moreover, the evidence confirms that reduction of stress can help in better management of glycaemic index [25]. Based on the evidence of the impact of stress on various chronic diseases including diabetes, it is important to screen for stress to improve diabetes management and also enhance the quality of life. Needless to add, there are many complexities involved in identification, measurement and management of stress. Since stress negatively impacts well-being and quality of life, a perspective on measurement of wellbeing could provide useful insights about the possible presence of stress. This approach could provide a useful screening 


\section{Current Research in Diabetes \& Obesity Journal}

mechanism to identify whether there is a need to probe further for depression or presence of stressors and so on.

The concept of wellbeing provides a perspective on the quality of life. This approach of measuring wellbeing provides a different paradigm for understanding the presence of stress (and also its impact on the wellbeing). The evidence supporting the use of WHO-5 to measure wellbeing and also to identify possible presence of depression makes a compelling case for the use of WHO-5 (i.e. measure wellbeing) instead of measuring stress. Based on the findings of wellbeing screening, further effort can focus on identification of emotional challenges including possible identification of stress related issues including sleep quality, trauma, depressive tendencies and so on. In other words, the simplicity of WHO-5 wellbeing screening and its ability to identify possible presence of stressors or emotional disturbances provides a useful framework to the physicians as compared to measurement of stress or mood or depression at the onset.

It would be useful to add that the health care professionals could still use stress surveys to identify environmental stress, personality profile tests to understand the resilience of the individual to the stressors and psychological surveys to measure the perception of stress. However, the approach of wellbeing measurement first appears to be more meaningful, easy to use and provides a powerful alternative to measurement of stress (including causes, perception and impact on the body and the mind).

\section{Recommendation}

The review recommends a simple, 5-questions based tool (WHO-5) developed by World Health Organization for screening for wellbeing and also for probing the presence of stressors in diabetes subjects. By measuring wellbeing using this tool, it is possible to understand the wellbeing of the individual and a lower score could be an indication for possible screening of depression or the need to understand the role of stress. Thus, by using this simple tool, it is possible to not only measure wellbeing but also identify possible presence of additional psychosocial stress or emotional vulnerability of the diabetes subject.

The simplicity (self-administered, easy to analyse, translation into about 30 languages), the availability of evidence (in psychology, chronic disease and diabetes subjects) further demonstrates the credibility of this tool in both clinical management of diabetes and its complications and also for scientific studies. Moreover, the increasing prevalence of diabetes (and other chronic disease) and resultant impact on cognitive functions provides a compelling framework to identify the presence of potential stressors (including lifestyle choices) and this is where WHO-5 provides a powerful framework for screening.

\section{References}

1. Trivedi Gunjan, Hemalatha Ramani, KV Ramani (2018) Chronic Diseases and Mind -Body Management, Part 1 - An Introduction (Technical Note), Indian Institute of Management.

2. (2019) International Diabetes Federation.

3. Moussavi S, Chatterji S, Verdes E, Tandon A, Patel V, et al. (2007) Depression, chronic diseases, and decrements in health: results from the World Health Surveys. Lancet 370(9590): 851-858.

4. Skaff MM, Mullan JT, Almeida DM, Hoffman L, Masharani U, et al. (2009) Daily negative mood affects fasting glucose in type 2 diabetes. Health Psychol 28(3): 265-272.

5. Cathy Lloyd, Julie Smith, Katie Weinger (2005) Stress and Diabetes: A Review of the Links. Diabetes Spectrum Apr 18(2): 121-127.

6. Skaff MM, Mullan JT, Almeida DM, Hoffman L, Masharani U, et al. (2009) Daily negative mood affects fasting glucose in type 2 diabetes. Health Psychol 28(3): 265-272.

7. Alexandros Heraclides, Tarani Chandola, Daniel R Witte, Eric J Brunner (2009) Psychosocial Stress at Work Doubles the Risk of Type 2 Diabetes in Middle-Aged Women. Diabetes Care Dec 32(12): 2230-2235.

8. Hackett RA, Steptoe A (2016) Psychosocial Factors in Diabetes and Cardiovascular Risk. Curr Cardiol Rep 18(10): 95.

9. Kalra S, Jena BN, Yeravdekar R (2018) Emotional and psychological needs of people with diabetes. Indian J Endocrinol Metab 22(5): 696704

10. Kopp MS, Thege BK, Balog P, Stauder A, Salavecz G, et al. (2010) Measures of stress in epidemiological research. J Psychosom Res 69(2): 211-225

11. (2019) WHO.

12. Sara Pinto, Laís Fumincelli, Alessandra Mazzo, Sílvia Caldeira José Carlos Martins (2017) Comfort, well-being and quality of life: Discussion of the differences and similarities among the concepts. Porto Biomedical Journal 2(1): 6-12.

13. Camfield L, Skevington SM (2008) On Subjective Well-being and Quality of Life. J Health Psychol 13(6): 764-775.

14.Zarbova Biserka, Karabeliova Sonya (2018) Stress and well-being Conference: $12^{\text {th }}$ International Conference Days of Applied Psychology 2016 "Contemporary Psychology and Practice. At: University of Nish, Serbia.

15. Lemyre L, Lalande-Markon MP (2009) Psychological Stress Measure (PSM-9): integration of an evidence-based approach to assessment, monitoring, and evaluation of stress in physical therapy practice. Physiother Theory Pract 25(5-6): 453-462.

16. Kopp MS, Thege BK, Balog P, Stauder A, Salavecz G, et al. (2010) Measures of stress in epidemiological research. J Psychosom Res 69(2): 211-225

17. Elissa S Epel, Alexandra D Crosswell, Stefanie E Mayer, Aric A Prather, George M Slavich, et al. (2018) More than a feeling: A unified view of stress measurement for population science, Frontiers in Neuroendocrinology 49: 146-169.

18. Psychiatric Research Unit, WHO Collaborating Center in Mental Health.

19. Goldberg DP, Blackwell B (1970) Psychiatric illness in general practice. A detailed study using a new method of case identification. Br Med J 1(5707): 439-443.

20. Topp CW, Østergaard SD, Søndergaard S, Bech P (2015) The WHO-5 Well-Being Index: A Systematic Review of the Literature. Psychother Psychosom 84(3): 167-176. 


\section{Current Research in Diabetes \& Obesity Journal}

21. de Wit M, Pouwer F, Gemke RJ, Delemarre-van de Waal HA, Snoek FJ (2007) Validation of the WHO-5 Well-Being Index in Adolescents with Type 1 Diabetes. Diabetes Care 30(8): 2003-2006.

22. Halliday JA, Hendrieckx C, Busija L, Browne JL, Nefs G, et al. (2017) Validation of the WHO-5 as a first-step screening instrument for depression in adults with diabetes: Results from Diabetes MILES Australia. Diabetes Res Clin Pract 132: 27-35.

23. Hochberg G, Pucheu S, Kleinebreil L, Halimi S, Fructuoso-Voisin C (2012) WHO-5, a tool focusing on psychological needs in patients with

his work is licensed under Creative Commons Attribution 4.0 Licens

DOl:10.19080/CRDOJ 2019.11.555820 diabetes: The French contribution to the DAWN study. Diabetes Metab 38(6): 515-522

24. Bech P, Olsen LR, Kjoller M, Rasmussen NK (2003) Measuring wellbeing rather than the absence of distress symptoms: a comparison of the SF-36 Mental Health subscale and the WHO-Five well-being scale. Int J Methods Psychiatr Res 12(2): 85-91.

25. Zamani-Alavijeh F, Araban M, Koohestani HR, Karimy M (2018) The effectiveness of stress management training on blood glucose control in patients with type 2 diabetes. Diabetol Metab Syndr 10: 39.

Your next submission with Juniper Publishers
will reach you the below assets
- Quality Editorial service
- Swift Peer Review
- Reprints availability
- E-prints Service
- Manuscript Podcast for convenient understanding
- Global attainment for your research
- Manuscript accessibility in different formats
( Pdf, E-pub, Full Text, Audio)
- Unceasing customer service
Track the below URL for one-step submission
- https://juniperpublishers.com/online-submission.php

